\title{
Constraining dynamical models with observational data
}

\author{
Jo Bovy ${ }^{1,2}$ \\ ${ }^{1}$ Institute for Advanced Study, Einstein Drive, Princeton, NJ 08540, USA \\ email: bovy@ias.edu \\ ${ }^{2}$ Hubble fellow
}

\begin{abstract}
The spatial distribution and kinematics of stars in the Milky Way are linked through the gravitational potential. Observations of the positions and velocities of stars can therefore be used to measure the mass distribution of the Milky Way. I review steady-state dynamical modeling approaches and illustrate their use in constraining the local matter distribution and the circular velocity curve from the kinematics of stellar tracers. In a few years, Gaia will increase the number of precise positions and velocities by multiple orders of magnitude. I describe some of the dynamical analyses that will be possible with the Gaia data and discuss some promising avenues for the optimal analysis of dynamical data.
\end{abstract}

Keywords. Galaxy: abundances, Galaxy: disk, Galaxy: fundamental parameters, Galaxy: general, Galaxy: kinematics and dynamics, Galaxy: stellar content, Galaxy: structure, ISM: kinematics and dynamics, solar neighborhood

\section{Introduction}

Historically, the mass content of galaxies and especially the presence and distribution of dark matter in galaxies has been primarily measured using the dynamics of tracers. The first evidence for the existence of dark matter came from measurements of the total mass in the solar neighborhood from the dynamics of stars perpendicular to the Galactic plane (Oort 1932) and from random motions of galaxies within a cluster that could not be explained by visible matter (Zwicky 1933). The rotation curve measurements of Rubin \& Ford (1970) and later work provided unequivocal evidence for the presence of dark matter in galaxies, since the flat rotation curves found at the outskirts of galaxies could not be explained by visible matter.

In the Milky Way, the initial measurements of the local density of matter of Oort have been refined using the precise HIPPARCOS data (e.g., Crézé et al. 1998; Holmberg \& Flynn 2000) and extended to constrain the total surface density to $\sim 1$ kpc (e.g., Kuijken \& Gilmore 1989b; Holmberg \& Flynn 2004). Current samples of stars are pushing these measurements to larger heights, such that robust constraints on the amount of dark matter can be obtained at heights where dark matter dominates the mass budget (heights $\gtrsim 1.5 \mathrm{kpc}$; Bovy \& Tremaine 2012) and such that the contributions from disk matter and dark matter can be separated (Zhang et al. 2013).

The upcoming Gaia space mission will allow for an unprecedented mapping of the mass distribution in the solar neighborhood and beyond using the dynamics of stellar tracers. Various groups are actively developing dynamical modeling techniques for optimal analysis of the Gaia data (see for example Binney et al. 2009 and Rix \& Bovy 2013). In this paper I briefly review modeling approaches for stellar tracers assumed to be in a steady state in an axisymmetric Galaxy (Sec. 2) and I discuss a few example analyses related to the Milky Way's rotation curve (Sec. 3) and the local surface density (Sec. 4). Section 5 
contains an outlook for Gaia analysis and a discussion of new avenues in steady-state dynamical modeling.

\section{Steady-state dynamical modeling}

The rotational and vertical motions of intermediate-age to old stars in the disk of the Milky Way may be assumed to a good approximation to be in a steady state. Memory of the initial conditions of the orbits of these stars will be washed out after a few dynamical times, such that all but the youngest stars have gone through multiple orbits around the Galactic center (period $\sim 220 \mathrm{Myr}$ ) and have performed many oscillations perpendicular to the disk (period $\sim 100 \mathrm{Myr}$; Binney \& Tremaine 2008). Therefore, the evolution of the stellar system can be described by the collisionless Boltzmann equation which relates the distribution function (DF) $f$ to the gravitational potential $\Phi$

$$
\frac{\mathrm{d} f}{\mathrm{~d} t} \equiv \frac{\partial f}{\partial t}+\mathbf{v} \cdot \frac{\partial f}{\partial \mathbf{x}}-\frac{\partial \Phi}{\partial \mathbf{x}} \cdot \frac{\partial f}{\partial \mathbf{v}}=0
$$

where the time derivative $\partial f / \partial t=0$ in a steady state.

Different steady-state dynamical modeling approaches differ in the manner in which the collisionless Boltzmann equation is solved simultaneously for the DF $f$ and the potential $\Phi$ given observational constraints. Of the techniques that I will not discuss here, Schwarzschild modeling is perhaps the most prominent (Schwarzschild 1979). Schwarzschild modeling proceeds by creating an orbit library for a given potential and matching the observed tracer density and velocities by applying weights to the orbits (subject to some smoothness constraint); the best-fitting potential-DF pair is then the preferred model. Schwarzschild modeling has been very successful in constraining the mass distributions of external galaxies (e.g., Rix et al. 1997) and in particular in measuring black hole masses in nearby galaxies (e.g., Gebhardt et al. 2000). However, Schwarzschild modeling has a few drawbacks that make it hard to apply in the MilkyWay/Gaia context, most prominent of which is that it is hard to apply to observations of individual stars due to the impractically large number of orbits that is required to remove numerical noise to match individual stars (McMillan \& Binney 2013).

A simple and popular approach is to calculate different velocity moments of the collisionless Boltzmann equation. This produces Jeans equations (Jeans 1915). Assuming that the system is axisymmetric and in a steady state, all time and azimuthal $(\phi)$ derivatives vanish; multiplying by the radial velocity $v_{R}$ and integrating over $v_{R}$ we obtain the radial Jeans equation

$$
F_{R}(R, Z)=-\frac{\partial \Phi(R, Z)}{\partial R}=\frac{1}{\nu} \frac{\partial\left(\nu \sigma_{R}^{2}\right)}{\partial R}+\frac{1}{\nu} \frac{\partial\left(\nu \sigma_{R Z}^{2}\right)}{\partial Z}+\frac{\sigma_{R}^{2}-\sigma_{T}^{2}-\bar{V}_{T}^{2}}{R},
$$

where $\nu$ is the tracer-density profile, $\sigma_{R}^{2}$ and $\sigma_{T}^{2}$ are the radial and tangential velocity dispersions squared, $\sigma_{R Z}^{2}$ is the off-diagonal radial-vertical entry of the dispersion-squared matrix, and $\bar{V}_{T}$ is the mean rotational velocity; all of these quantities are functions of $R$ and $Z$. Similarly, multiplying the collisionless Boltzmann equation by $v_{Z}$ and integrating over $v_{Z}$ we get the vertical Jeans equation

$$
F_{Z}(R, Z)=-\frac{\partial \Phi(R, Z)}{\partial Z}=\frac{1}{\nu} \frac{\partial\left(\nu \sigma_{Z}^{2}\right)}{\partial Z}+\frac{1}{R \nu} \frac{\partial\left(R \nu \sigma_{R Z}^{2}\right)}{\partial R}
$$

where $\sigma_{Z}$ is the vertical velocity dispersion.

The Jeans equations (2.2-2.3) relate the spatial and kinematic properties of a tracer population (right-hand sides) to the forces due to the mass distribution (left-hand sides). 
Thus, by measuring the spatial distribution and kinematics of a tracer population they allow the mass distribution to be inferred. In Section 3 we will apply the radial Jeans equation (2.2) to model the kinematics of a set of stellar tracers in the plane of the Milky Way's disk and thus measure the rotation curve. In Section 4 we will use the vertical Jeans equation (2.3) to model the vertical dynamics of stars near the Sun.

A different approach starting from the collisionless Boltzmann equation is to use the Jeans theorem (Binney \& Tremaine 2008) which states that any steady-state solution of the collisionless Boltzmann equation can only depend on the phase-space coordinates through integrals of the motion. The strong Jeans theorem further specifies that in the case in which most orbits are regular with non-resonant frequencies, the DF can be assumed to be a function of three isolating integrals only. Therefore, we can model stellar populations by fitting the six-dimensional phase-space distribution using a function of three variables only. This still leaves a large amount of freedom and in practice one typically assumes a parameterized form for the DF in terms of integrals of the motion, although more general techniques have been developed (e.g., Bovy et al. 2010; Magorrian 2013). This is the approach taken in Kuijken \& Gilmore (1989ab). I will discuss this approach further in Section 5 when considering future modeling approaches for Gaia.

\section{The Milky Way's rotation curve}

Because the radial force is related to the circular velocity as $R F_{R}(R)=-V_{c}^{2}(R)$, we can use equation (2.2) to constrain the circular velocity given measurements of the radial and vertical derivatives of the density $\nu$, the radial and tangential velocity dispersions $\sigma_{R}$ and $\sigma_{T}$, the cross-term $\sigma_{R Z}^{2}$, and the mean rotational velocity $\bar{V}_{T}$ of a population of tracers. When observing in the plane of the Milky Way, we can assume that the vertical density gradient and the cross-term $\sigma_{R Z}^{2}$ are zero because of symmetry and we find that

$$
V_{c}^{2}=\bar{V}_{T}^{2}-\frac{R}{\nu} \frac{\partial\left(\nu \sigma_{R}^{2}\right)}{\partial R}-\sigma_{R}^{2}+\sigma_{T}^{2} .
$$

Therefore, the circular velocity squared is equal to the mean rotational velocity squared minus a small correction proportional to the dispersion of the tracer population; this correction is known as the asymmetric drift.

We can compare the measurement of the circular velocity based on a kinematicallywarm tracer population (i.e., a population with non-negligible velocity dispersion) to that based on kinematically-cold ISM tracers. Since in an axisymmetric model for the Galaxy cold tracers move on circular orbits, their rotational velocities in principle provide a direct measurement of the circular velocity. Thus, cold tracers provide perhaps the most direct mapping of the circular velocity possible. Historically, this measurement has been primarily performed using $\mathrm{HI}$ and $\mathrm{CO}$ emission, the line-of-sight velocity of which has a maximum at the tangent point for $-90^{\circ} \leqslant l \leqslant 90^{\circ}$; the tangent point is the location along the line-of-sight that is the closest to the Galactic center. At this point the line-of-sight velocity $v_{\mathrm{LSR}}$ is given by

$$
v_{\mathrm{LSR}}=R_{0} \sin l\left(\Omega-\Omega_{0}\right),
$$

where $\Omega=V_{c} / R$. For the purpose of comparison with measurements based on kinematically-warm tracers, the most important properties of the tangent-point method for mapping the rotation curve are:

- A value for $V_{c}\left(R_{0}\right)$ has to be assumed to transform $v_{\mathrm{LSR}}$ into a measurement of $V_{c}(R)$. Thus, $V_{c}\left(R_{0}\right)$ needs to be measured using a different method. 
- The measured maximum velocity $v_{\mathrm{LSR}}$ is directly related to the circular velocity, for an assumed value of the circular velocity $V_{c}\left(R_{0}\right)$ at $R_{0}$. Without an independent measurement of $V_{c}\left(R_{0}\right)$, the tangent-point method is insensitive to solid-body rotation.

- For any given $l$ the measurement is only possible at a single $R$. Therefore, bumps and wiggles in the tangent-point curve cannot be unambiguously ascribed to features in the rotation curve or the influence of non-axisymmetric streaming motions.

- Kinematically-cold dynamical tracers are more sensitive to non-axisymmetric perturbations than kinematically-warmer populations (e.g., Lin et al. 1969).

We can contrast this with the properties of kinematically-warm stellar tracers:

- The circular velocity can be measured at any $R$, including $R>R_{0}$ and $R=R_{0}$. Therefore, there is no need for an independent measurement of $V_{c}\left(R_{0}\right)$.

- Because of the non-zero velocity dispersion, the radial density profile and the value and radial profile of the velocity dispersions is required in equation (3.1). With limited data this typically involves modeling these dispersions using parameterized functions(see below).

- $V_{c}(R)$ can be measured at any azimuthal angle, so a two-dimensional mapping of the effect of non-axisymmetry can in principle be disentangled from features in the radial profile of the rotation curve.

- Kinematically-warm tracers are less sensitive to non-axisymmetric perturbations.

As an example of a measurement of the circular velocity curve from the kinematics of kinematically-warm stellar tracers, I consider data from SDSS-III/Apache Point Observatory Galactic Evolution Experiment (APOGEE). APOGEE is a near-infrared ( $H$-band; 1.51 to $1.70 \mu \mathrm{m}$ ), high-resolution $(\mathrm{R} \approx 22,500)$, spectroscopic survey, targeting primarily red giants in all Galactic environments, with emphasis on the disk and the bulge (S. R. M. Majewski. et al. 2013, in preparation). I refer the reader to Bovy et al. (2012b) for more information about the APOGEE data set as well as the full details of the dynamical analysis; only the most interesting aspects from the standpoint of constraining dynamical models from observational data are discussed here. The APOGEE sample used here covers $30^{\circ} \leqslant l \leqslant 210^{\circ}, d \lesssim 10 \mathrm{kpc}$, and has extremely precise velocity measurements (uncertainties $\sim 100 \mathrm{~m} \mathrm{~s}^{-1}$ ).

APOGEE as a spectroscopic survey measures only the heliocentric line-of-sight velocity component $V_{\text {los }}^{\text {helio }}$ and we do not use any proper motion data. The Galactocentric lineof-sight velocity $V_{\text {los }}^{\text {gal }}$ depends on the Sun's peculiar motion in the Galactocentric frame

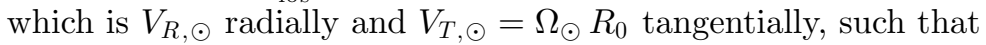

$$
V_{\mathrm{los}}^{\mathrm{gal}}=V_{\mathrm{los}}^{\text {helio }}-V_{R, \odot} \cos l+\Omega_{\odot} R_{0} \sin l,
$$

We model the kinematics of the stellar tracers as follows: (a) The velocity distribution at any given position is assumed to be a bi-axial Gaussian with a fixed axis ratio that is a free parameter in the fit, (b) the mean radial velocity is zero under the assumption 
of axisymmetry and the mean rotational velocity is calculated from the circular velocity using the correction from equation (3.1), (c) the radial dispersion $\sigma_{R}(R)$ is assumed to have an exponential radial profile with a scale length that is a free parameter. We further assume that the tracer density has a scale length of $3 \mathrm{kpc}$, although this does not influence the result much. Therefore, the DF is characterized by three free parameters: (a) the radial dispersion at $R_{0}$, (b) the dispersion scale length, and (c) the ratio of the radial and tangential dispersions.

The mean line-of-sight velocity in the heliocentric frame is given by

$$
\bar{V}_{\mathrm{los}}=\bar{V}_{T} \sin (\phi+l)+V_{R, \odot} \cos l-\Omega_{\odot} R_{0} \sin l .
$$

The fact that the APOGEE sample goes out to large $(\sim 10 \mathrm{kpc})$ distances at $\sin l \neq$ 0 means that the azimuthal angle $\phi$ is significantly non-zero for a large part of the sample. This fact allows us to disentangle the Sun's peculiar motion $\left(V_{R, \odot}, \Omega_{\odot} R_{0}\right)$ with its $(\cos l, \sin l)$ dependence from the circular velocity with its $\sin (\phi+l)$ dependence.

The results of this dynamical model of the APOGEE data are the following:

- $V_{c}=218 \pm 6 \mathrm{~km} \mathrm{~s}^{-1}$ when using a flat-rotation-curve model; the best-fit value for $V_{c}\left(R_{0}\right)$ is the same when considering a power-law or linear model for the rotation curve, but the lower-limit of the 1 sigma range becomes $200 \mathrm{~km} \mathrm{~s}^{-1}$.

- The best-fit rotation curve is flat: for a power-law model $V_{c}(R) / V_{c}\left(R_{0}\right)=$ $\left(R / R_{0}\right)^{\beta}, \beta=0.01_{-0.10}^{+0.01}$.

- The Sun's peculiar motion is measured independently from $V_{c}: V_{R, \odot}=$ $-10.5 \mathrm{~km} \mathrm{~s}^{-1}, V_{T, \odot}=242 \mathrm{~km} \mathrm{~s}^{-1}$.

These results are close to the IAU recommended value for $V_{c}$ of $220 \mathrm{~km} \mathrm{~s}^{-1}$. Marginalizing over a host of possible systematics, our results are that $V_{c}<235 \mathrm{~km} \mathrm{~s}^{-1}$ at $99 \%$ confidence and as such are strongly inconsistent with a much higher value for $V_{c}$.

\section{The vertical mass distribution near the Sun}

The vertical mass distribution near the plane of the Galactic disk can be measured using the vertical dynamics of stellar tracers. This approach goes back to Oort (1932) and has been applied many times since its first inception (see references in the introduction). Our starting point is the vertical Jeans equation (2.3). While it is in principle possible to measure the cross-term $\sigma_{R Z}^{2}$ and how it varies with $R$, in practice this is difficult. It is only important at large distances from the mid-plane (e.g., Bovy \& Tremaine 2012) and when staying close to the mid-plane $(\lesssim 1.5 \mathrm{kpc})$ it can be safely set to zero (see discussion in Zhang et al. 2013). The vertical Jeans equation then becomes a purely vertical equation, i.e., all relevant gradients are vertical. The vertical force law can then be measured from the vertical density distribution and kinematics of stars.

We can obtain the mass density from the gravitational potential using the Poisson equation. In cylindrical coordinates this can be written as

$$
\Sigma(R, Z)=-\frac{1}{2 \pi G}\left[\int_{0}^{Z} \mathrm{~d} z \frac{1}{R} \frac{\partial\left(R F_{R}\right)}{\partial R}+F_{Z}(R, Z)\right] .
$$

Because $R F_{R}=-V_{c}^{2}$, the integrand in the term on the right-hand side is proportional to the derivative of the circular velocity, which is known to be small. Moreover, in any reasonable model for the density distribution near the mid-plane, the integral can be 
accurately approximated assuming that the integrand is constant at its value in the plane, that is, the first term can be approximated as $\left(A^{2}-B^{2}\right)|Z|$, where $A$ and $B$ are Oort constants (Kuijken \& Gilmore 1989), even at multiple kpc from the mid-plane (Bovy \& Tremaine 2012). Measurements of the circular velocity curve and Oort constants, such as that described in Section 3 above, show that this term is negligible compared to the contribution from the vertical force.

When we neglect the first term in square brackets in equation (4.1) and combine it with the vertical Jeans equation we find that the vertical mass distribution can be measured using the density and vertical velocity dispersion of a set of tracers. This method has been applied numerous times in the past. We focus here on some recent progress.

Previous analyses of the vertical mass distribution near the Sun have been hampered by a lack of good distances, as HIPPARCOS did not measure precise distances at $\sim 1 \mathrm{kpc}$ from the Sun. Recently, the SDSS/SEGUE has taken spectra of several well-defined, homogeneous samples of disk stars, e.g., the G-type and K-type dwarf samples (Yanny et al. 2009). The spectra combined with the homogeneous five band SDSS photometry give spectrophotometric distances good to $\sim 12 \%$, which combined with proper motions and line-of-sight velocities provides full $6 \mathrm{D}$ phase-space information for tens of thousands of stars. The spectra allow for metallicities $[\mathrm{Fe} / \mathrm{H}]$ and alpha-enhancements $[\alpha / \mathrm{Fe}]$ to be measured for these main-sequence samples characterized by a narrow range of effective temperatures; these abundance measurements allow us to group chemically similar stars, which have been shown to have simple spatial and kinematic distributions (e.g., Bovy et al. 2012a).

In Zhang et al. (2013) we applied this methodology to jointly model the spatial distribution and kinematics of three abundance-selected sub-populations of K-type dwarfs: a sample of stars with solar $[\mathrm{Fe} / \mathrm{H}]$ and $[\alpha / \mathrm{Fe}]$, a sample with intermediate abundances, and a sample of metal-poor, $[\alpha / \mathrm{Fe}]$-enhanced stars. These three samples are modeled using independent DFs (characterized by a scale height and vertical velocity dispersion) and a single model for the vertical force law. The spatial density is carefully corrected for the SEGUE sampling density. The vertical force law is modeled using the form proposed by Kuijken \& Gilmore (1989a): this force law is characterized by the contribution from the disk, its scale height, and the contribution from the halo.

The SEGUE K-type dwarfs are found to tightly constrain the vertical mass distribution between $300 \mathrm{pc}$ and $1.2 \mathrm{kpc}$ and they provide the following measurements of the disk and halo contributions to the local mass budget:

- The total surface density to $1 \mathrm{kpc}: \Sigma(|Z| \leqslant 1 \mathrm{kpc})=67 \pm 6 M_{\odot} \mathrm{pc}^{-2}$.

- The contribution from the disk, $\Sigma_{\text {disk }}=42 \pm 5 M_{\odot} \mathrm{pc}^{-2}$, and the disk scale height $\lesssim 300$ pc.

- The local dark matter density: $\rho_{\mathrm{DM}}=0.0065 \pm 0.0023 M_{\odot} \mathrm{pc}^{-3}$.

The last number is in good agreement with the results from a similar recent analysis based on the kinematics of stars at large heights $(1.5 \mathrm{kpc} \leqslant|Z| \leqslant 4.5 \mathrm{kpc}$; Bovy \& Tremaine 2012).

\section{Discussion and outlook}

The Gaia satellite that will soon be launched will measure parallaxes, proper motions, and line-of-sight velocities of $\sim 10^{9}$ stars down to $G=20 \mathrm{mag}$. Thus for a large number 
of stars we will obtain precise $6 \mathrm{D}$ phase-space coordinates out to multiple kpc. This will allow dynamical analyses such as those described in this paper to be carried out with larger samples of stars with geometric distances and homogeneous proper motions in addition to line-of-sight velocities that are of somewhat lower quality than the spectroscopic velocities obtained by SEGUE and APOGEE, but that are still good enough in a Galactic-dynamics context.

In particular, with Gaia it will be possible to analyze the vertical dynamics of stellar tracers as we did in Section 4 away from the solar cylinder. As the vertical dynamics directly measures the mass distribution near the disk of the Milky Way, this will allow the dynamical measurement of the radial profile of the mass distribution and its decomposition in disk and halo contributions. While near the Sun the phase-space measurements will be precise enough to allow the forces to be directly mapped by using the Jeans equations, going to larger distances to measure the large-scale mass distribution will require going to the low signal-to-noise regime of the survey where the effect of distance and velocity uncertainties as well as missing line-of-sight velocities (which will only be obtained down to $G \approx 17$ and this only at the end of the survey). Therefore, dynamical modeling approaches that can deal with uncertainties, missing phase-space dimensions, and outliers will still be necessary to make the most interesting inferences based on the Gaia data.

A promising avenue in this respect is presented in the work of Binney (2010) and papers following on that. The basic assumption in this work is again that disk populations can be modeled as being in a steady-state and that a reasonable starting point for dynamical models is an axisymmetric approximation before allowing non-axisymmetric, timedependent gravitational potentials. Rather than using the Jeans equations, the strong Jeans theorem is invoked to posit that the $6 \mathrm{D} \mathrm{DF}$ of a disk population can be modeled as a super-position of simple 3-action DFs, viz., quasi-isothermal DFs (Binney 2010, with improvements by Binney \& McMillan 2011). These quasi-isothermal DFs are a family of simple, analytic DF models that are parameterized by the radial density profile and the vertical and radial velocity dispersion and its dependence on $R$. Binney (2010) and Binney (2012) have shown that a super-position of these DFs leads to a good fit to local data from the Geneva-Copenhagen survey and to the density and kinematics of stars at $\sim 1 \mathrm{kpc}$.

Because the quasi-isothermal DF model provides a generative model-i.e., it can be used to generate a set of mock data drawn from the DF for any given potential - it can be used straightforwardly in a likelihood-based dynamical modeling approach. The likelihood of a model for the tracer DF and the gravitational potential is given by the quasi-isothermal evaluated at the position of the data points. In a likelihood approach, accounting for observational uncertainties, missing data, and selection effects can be achieved through marginalization and projection of the model into the space of observables. In the context of the quasi-isothermal DF this is presented in McMillan \& Binney (2013) and Ting et al. (2013).

While the approach of Binney (2012) and McMillan \& Binney (2013) is to model the disk as a super-position of a large number of quasi-isothermal DFs, it has recently become clear that measurements of the elemental abundances of stars can be used to simplify the DF model. In particular, Ting et al. (2013) found, based on the results of Bovy et al. (2012a), that when stars within a few kpc from the Sun are grouped into populations with very similar $[\mathrm{Fe} / \mathrm{H}]$ and $[\alpha / \mathrm{Fe}]$ (bins with width $0.1 \mathrm{dex}$ in $[\mathrm{Fe} / \mathrm{H}]$ and 0.05 dex in $[\alpha / \mathrm{Fe}])$ their spatial density and kinematics is well represented by a single quasi-isothermal DF. This is a strong simplification over the super-position approach and 
it allows dynamical modeling to proceed without assumptions about the DF parameters on (effectively) hidden parameters such as age as in Binney (2012).

Ting et al. (2013) showed with mock data that with a few thousand stellar tracers a single quasi-isothermal DF model can constrain the amplitude of the potential to one percent and the flattening to a few percent, using observations out to a few kpc from the Sun. Further development of this technique is necessary for Gaia data analysis. In particular, as Gaia will not measure $[\alpha / \mathrm{Fe}]$, it remains to be tested whether a cruder abundance-selection based on $[\mathrm{Fe} / \mathrm{H}]$ only - which Gaia will be able to measure for many stars - is sufficient to allow for the Ansatz of a single quasi-isothermal DF.

We are currently in the process of applying the technique described by Ting et al. (2013) to the SEGUE G dwarf data in order to measure the surface density at $\sim 1 \mathrm{kpc}$ between $5 \mathrm{kpc}$ and $9 \mathrm{kpc}$ (Bovy \& Rix 2013, in preparation).

Despite the advantages of the direct DF modeling approach as compared to the Jeans modeling described in Sections 3 and 4, it has a few limitations that need to be addressed before it can be applied to the Gaia data:

- The calculation of the actions and the effective volume of the survey (required to normalize the DF over the survey volume) is slow and requires a large number of DF evaluations. It is currently extremely challenging to make the computation fast enough to scan through the large number of DF and potential parameters that would be required for the full Gaia data analysis.

- The action-based DF approach requires a full analytic 3D model for the Milky Way's gravitational potential, to allow for the quick evaluation of the actions. It remains to be tested how sensitive any dynamical analyses are to the specific details of the gravitational potential and the analytic form of the DF, especially if because of the computational complexity discussed above not all of the necessary potential or DF parameters can be varied during the dynamical modeling.

- In particular, the assumption of axisymmetry and regular, non-resonant orbits needs to be tested in detail for all steady-state, axisymmetric dynamical modeling methods to account for the fact that the Milky Way's potential likely has significant non-axisymmetric, time-dependent contributions such as spiral arms, a bar, a warp, and other asymmetries routinely seen in $N$-body simulations of galaxies and for which there is significant evidence from the kinematics of stars and gas (e.g., Blitz \& Spergel 1991; Dehnen 1998). As mentioned above, the effects of non-axisymmetry are larger for the kinematics in the plane of the Galaxy than for the vertical oscillations of stars. The effects and potential biases this induces, especially for DF-based approaches that couple the vertical and radial oscillations, need to be carefully assessed.

The Gaia data that will be available in a few years hold the promise of greatly improving our knowledge of the mass distribution in the inner Milky Way and beyond. As most disk stars are dynamically old (i.e., they have orbited for many dynamical times), modeling disk populations as being in a steady state is a fruitful and well-developed approach for modeling the dynamics and thus the mass distribution in the Milky Way close to the plane of the disk. The scale and complexity of the Gaia data require further development of dynamical modeling approaches, especially those that can deal with observational uncertainties, missing data, selection biases, and modeling imperfections. 
The methods described in this paper are well-situated for providing such a methodology and the extensions described in this Section provide a clear path toward the optimal analysis of the Gaia data.

\section{Acknowledgments}

J.B. was supported by NASA through Hubble Fellowship grant HST-HF-51285.01 from the Space Telescope Science Institute, which is operated by the Association of Universities for Research in Astronomy, Incorporated, under NASA contract NAS5-26555.

\section{References}

Adibekyan, V. Zh., Sousa, S. G., Santos, N. C., et al. 2012, Astron. Astrophys., 545, A32

Binney, J. J. 2010, Mon. Not. Roy. Astron. Soc., 401, 2318

Binney, J. J. 2012, Mon. Not. Roy. Astron. Soc., 426, 1328

Binney, J. J. \& McMillan, P. 2011, Mon. Not. Roy. Astron. Soc., 413, 1889

Binney, J. \& Tremaine, S., 2008, Galactic Dynamics: Second Edition (Princeton University Press)

Binney, J. J., et al. 2009, Proceedings of Joint Discussion 5 at IAU XXVII, Rio de Janeiro, arXiv:0911.2661

Blitz, L. \& Spergel, D. N. 1991, Astrophys. J., 379, 631

Bovy, J., Murray, I., \& Hogg, D. W. 2010, Astrophys. J., 711, 1157

Bovy, J., Rix, H.-W., Liu, C., et al. 2012a, Astrophys. J., 753, 148

Bovy, J. \& Tremaine, S. 2012, Astrophys. J., 756, 89

Bovy, J., Allende Prieto, C., Beers, T. C., et al. 2012b, Astrophys. J., 759, 131

Creze, M., Chereul, E., Bienaymé, O., \& Pichon, C. 1998, Astron. Astrophys., 329, 920

Dehnen, W. 1998, Astron. J., 115, 2384

Gebhardt, K., Richstone, D., Kormendy, J., et al. 2000, Astrophys. J., 119, 1157

Fuhrmann, K. 2011, Mon. Not. Roy. Astron. Soc., 414, 2893

Holmberg, J. \& Flynn, C. 2000, Mon. Not. Roy. Astron. Soc., 313, 209

Holmberg, J. \& Flynn, C. 2004, Mon. Not. Roy. Astron. Soc., 352, 440

Jeans, J. H. 1915, Mon. Not. Roy. Astron. Soc., 76, 70

Kuijken, K. \& Gilmore, G. 1989a, Mon. Not. Roy. Astron. Soc., 239, 571

Kuijken, K. \& Gilmore, G. 1989b, Mon. Not. Roy. Astron. Soc., 239, 605

Lin, C. C., Yuan, C., \& Shu, F. H. 1969, Astrophys. J., 155, 721

Magorrian, J. 2013, Mon. Not. Roy. Astron. Soc., submitted

McMillan, P. \& Binney, J. J. 2013, Mon. Not. Roy. Astron. Soc., in press

Oort, J. H. 1932, Bull. Astron. Inst. Neth., 6, 249

Rix, H.-W. \& Bovy, J. 2013, Astron. Astrophys. Rev., 21, 61

Rix, H.-W., de Zeeuw, P. T., Cretton, N., van der Marel, R. P., \& Carollo, C. M. 1997, Astrophys. J., 488, 702

Rubin, V. C. \& Ford, W. K. Jr. 1970, Astrophys. J., 159, 379

Schwarzschild, M. 1979, Astrophys. J., 232, 236

Stepanishchev, A. S. \& Bobylev, V. V. 2013, Ast. L., 39, 185

Ting, Y.-S., Rix, H.-W., Bovy,J., \& van de Ven, G. 2013, Mon. Not. Roy. Astron. Soc., in press

Yanny, B., Rockosi, C., Newberg, H. J., et al. 2009, Astron. J., 137, 4377

Zhang, L., Rix, H.-W., van de Ven, G, Bovy, J., Liu, C., \& Zhao, G. 2013, Astrophys. J., in press

Zwicky, F. 1933, Helv. Phys. Acta 6, 110

\section{Discussion}

Poul ERIK Nissen: I am puzzled that you from the SDSS data find a disk population with velocity dispersion and $[\alpha / \mathrm{Fe}]$ intermediate between thin-disk and thick-disk stars. High-resolution spectroscopic studies of volume-limited samples (Fuhrmann 2011 at $d<$ 
$20 \mathrm{kpc}$; Adibekyan et al. 2012 at $d<60 \mathrm{kpc}$ ) show a gap in the $[\alpha / \mathrm{Fe}]$ where you find an intermediate disk population.

Jo Bovy: This is indeed puzzling, but we note that the volume sampled by the highresolution samples is much smaller than that observed by the SDSS survey $\left(\sim 10^{-3} \mathrm{kpc}^{3}\right.$ vs. $\sim 10 \mathrm{kpc}^{3}$ ). Surveys such as APOGEE and Gaia-ESO will extend the high-resolution samples to much larger distances from the Sun and therefore they will be able to resolve this question. Based on the isothermal kinematics of the intermediate $[\alpha / \mathrm{Fe}]$ populations it is highly improbable that the intermediate populations are not real.

MiCHAEL FEAST: Is your data consistent with a falling rotation curve at the solar position (as seen in VLBI maser and HIPPARCOS Cepheid data)?

Jo Bovy: The inferred rotation curve in the APOGEE circular velocity analysis is fully consistent with the rotation curves found by the HIPPARCOS Cepheids and the VLBI observations of masers.

Michael FeAst: Stepanishchev and Bobylev (2013) find that correction for bias reduces the angular velocity of rotation, suggesting $V_{c}$ is nearer $220 \mathrm{~km} \mathrm{~s}^{-1}$.

Jo Bovy: That is interesting. Their assumption that the masers lie in an exponential disk with a scale length of $3 \mathrm{kpc}$ is likely wrong. The scale length is probably larger, and there also appears to be a hole in the central $4 \mathrm{kpc}$ in the ISM. If that is the case, then the Lutz-Kelker corrections that they compute for their sources closest to the Galactic center (which are the only ones for which the bias matters, see their Fig. 1) are probably wrong. 